\section{Optimal Noise Filters in High-Resolution Electron Microscopy}

\author{
Kazuo Ishizuka, Paul H. C. Eilers ${ }^{\star}$ and Toshihiro Kogure** \\ HREM Research Inc., Higashimatsuyama, Japan \\ * Utrecht University, Utrecht, The Netherlands \\ ** University of Tokyo, Tokyo, Japan \\ ishizuka@hremresearch.com
}

\section{Introduction}

Most of the specimens for high-resolution electron microscopy have amorphous surface layers due to contamination during observation and/or damaged surface layers during specimen preparation. Moreover, many specimens are radiation sensitive, and a part of the specimen easily becomes amorphous during the observation. These amorphous materials make clear observation of crystal structure difficult. A periodic structure may be extracted by simply using a periodic mask in Fourier space. However, this kind of mask often introduces a periodic feature in addition to the crystal structure. To reduce such artifacts a Wiener filter or an average background subtraction filter has been discussed $[1,2]$. However, these filters do not work for non-ideal crystals, such as cylindrical crystals and nano-crystals, where a translational periodicity is limited to the order of nano-meter. In this report we improve these filters by introducing new ways to estimate a contribution from the amorphous materials.
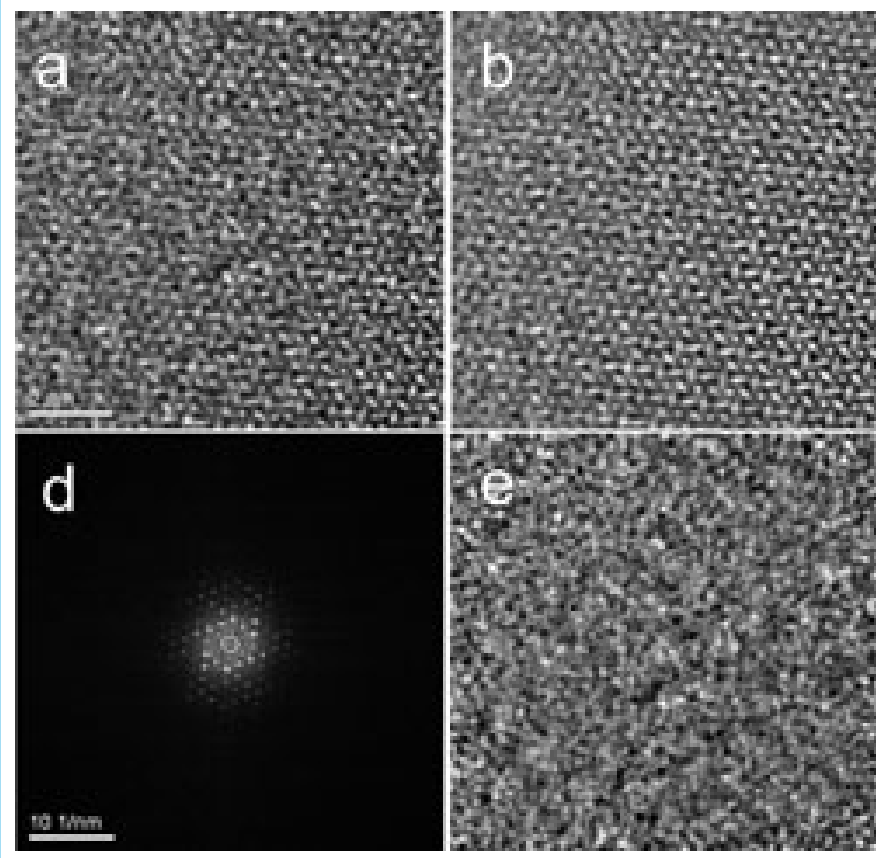

Figure 1 Original image of $\mathrm{Si}_{3} \mathrm{~N}_{4}(a)$ and its Fourier transform $(d)$. Image size is $512 x 512$ pixels. $(b)$ and (c): Results from Wiener filters based on radial and periodic mask backgrounds, respectively. (e) and (f): Differences between the original and filtered images shown in (b) and (c), respectively. Both Wiener filters works well for this kind of periodic image, and thus differences do not show any residual periodic structure.

\section{Wiener filter}

Figure 1 shows a part of $\mathrm{Si}_{3} \mathrm{~N}_{4}$ image (a) and its Fourier transform (d). The image used here is made available by C. Kisielowski. Here, the crystal structure degrades towards the top left part of the image, and the Fourier transform shows concentric rings corresponding to a diffractogram of amorphous material. This ring pattern comes from a non-periodic part in the image and prob- ably from amorphous surface layers. (The Fourier tra

more periodic region (not shown here) also shows a similar ring pattern.) The Fourier transform $F_{o}$ of an observed image may be written as a sum of a true signal $F_{c}$ originating in the crystal part and a non-periodic background $F_{b}$ from a non-crystal part, and thus $F_{o}=F_{c}+F_{b}$. The true signal $F_{c}$ is confined to diffraction spots and is usually strong, while the non-periodic background $F_{b}$ distributes more evenly.

The Wiener filter seeks a solution that minimizes the summed square difference between the true signal $F_{c}$ and its estimate $\hat{F}_{w}$ :

$$
\sum\left|\hat{F}_{W}-F_{c}\right|^{2}=\sum\left|M_{W} F_{o}-F_{c}\right|^{2} \Rightarrow \text { minimum },
$$

where we approximate the estimate with a product of a mask $M_{w}$ and the Fourier transform of an observed image: $M_{w} F_{o}$. When we assume that the signal and noise are uncorrelated, the appropriate solution for $M_{w}$ is given by

$$
M_{W}=\left|F_{c}\right|^{2} /\left|F_{o}\right|^{2} \approx\left(\left|F_{o}\right|^{2}-\left|F_{b}\right|^{2}\right) /\left|F_{o}\right|^{2},
$$

where we use $\left|F_{o}\right|^{2} \approx\left|F_{c}\right|^{2}+\left|F_{b}\right|^{2}$. Thus, we have an applicable form of the Wiener filter given by

$$
\hat{F}_{W}=M_{W} F_{o} \approx \frac{\left|F_{o}\right|^{2}-\left|\hat{F}_{b}\right|^{2}}{\left|F_{o}\right|^{2}} F_{o}
$$

where $\left|\hat{F}_{b}\right|$ is an estimate of a non-periodic background. Here, $M_{w}$

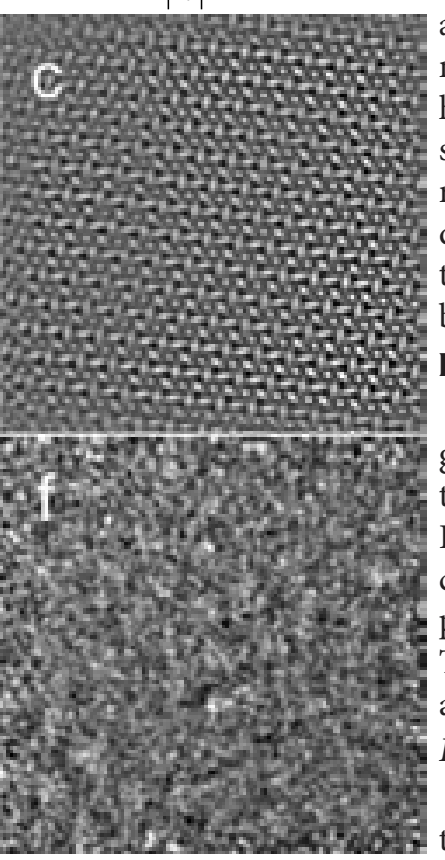
and thus $\hat{F}_{w}$ is set to zero, if $\left|F_{o}\right|-\left|\hat{F}_{b}\right| \leq 0$ namely, the estimated background is higher than the observed value. This situation often happens at a background region in Fourier space due to the random nature of the background. Thus, this is one of the mechanisms that may be used to reduce random noise.

\section{Background estimation}

In order to use the Wiener filter given by Eq. (1) we have to estimate the background contribution $\left|\hat{F}_{b}\right|$ in Fourier space. Here, we assume that the contribution from amorphous (nonperiodic) materials varies slowly $[1,2]$. Then, the background may be estimated as explained below: Radial Background

The background is normally estimated as a rotational average of the Fourier transform of the whole image. Here, an average is taken after excluding strong intensities corresponding to Bragg diffraction originating in the periodic structure $[1,2]$.

\section{Two-Dimensional Background}

However, a radial background will not work, when the structure information appears at the same distance from the origin in Fourier space. Thus, we developed a novel approach based on P-spline fitting [3] to estimate a smoothed two-dimensional background in Fourier space. 


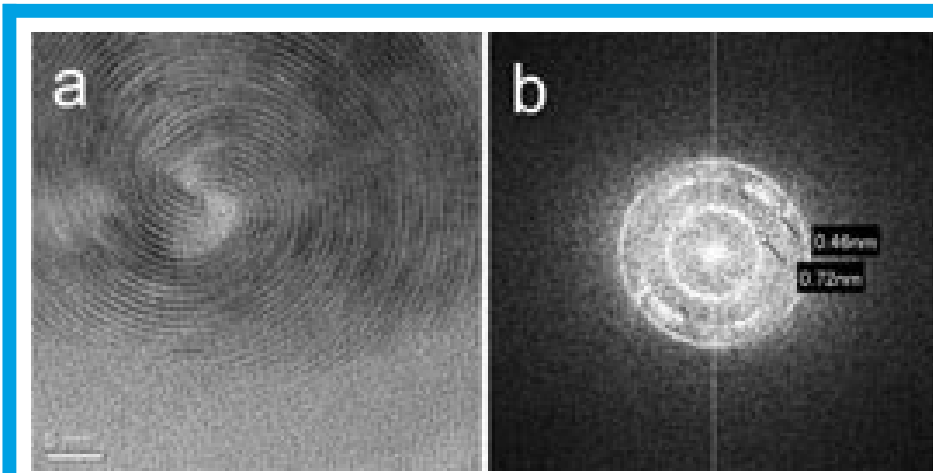

Figure 2 Original image of crysotile (a) and its Fourier transform (b). An image size is $512 \times 512$ pixel. Here, $0.72 \mathrm{~nm}$ corresponds to an interlayer spacing, while $0.46 \mathrm{~nm}$ a repeat distance of structural blocks within each layer.

\section{Local Two-Dimensional Background}

When a periodic structure becomes small in size, its orientation changes locally. Then, the background estimated for the whole image may not be adequate. Thus, a two-dimensional background in Fourier space is locally estimated for each small image area by dividing a whole image into many small areas.
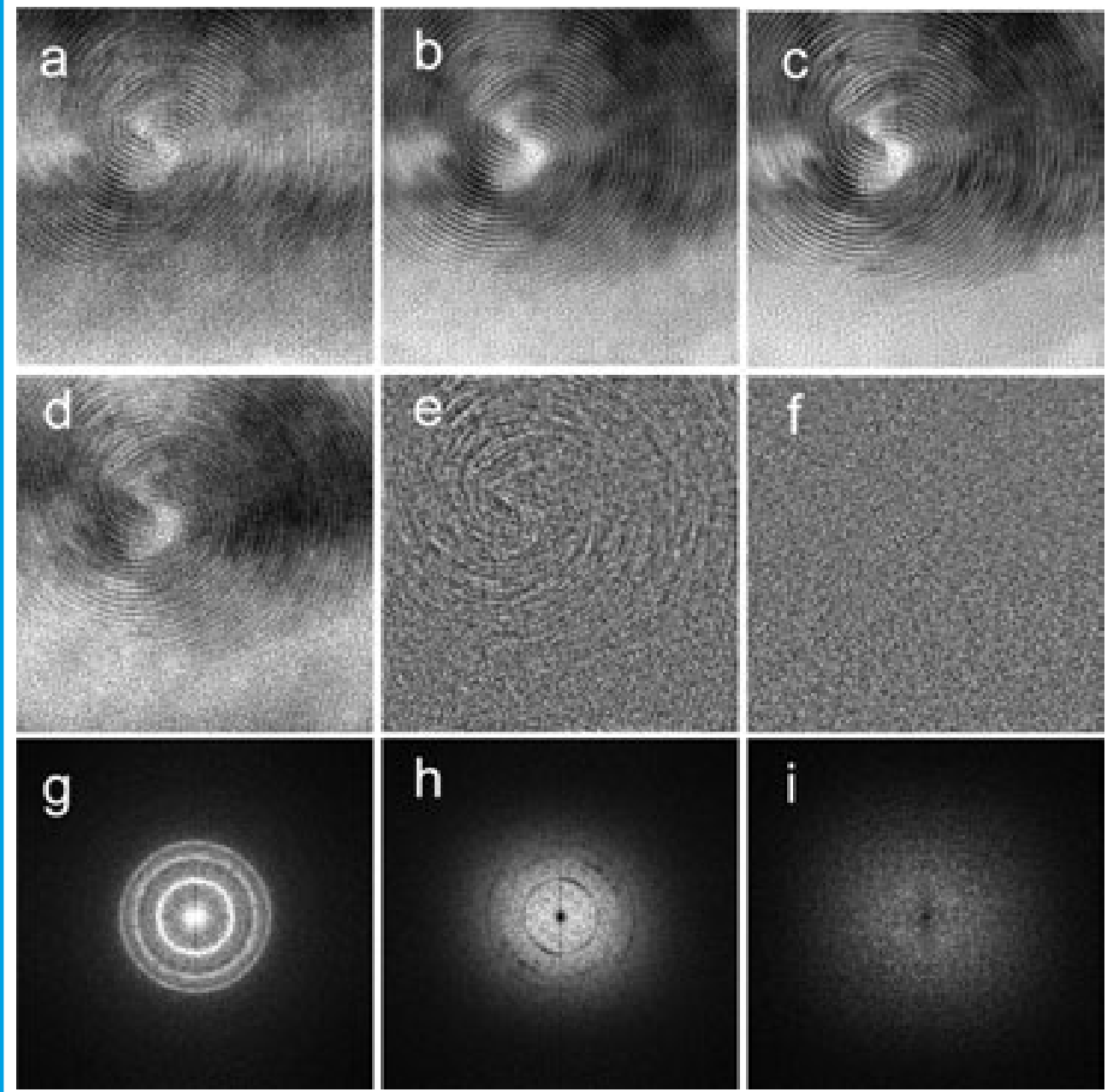

Figure 3 (a) to (c): Three Wiener filtered images of crysotile using a radial background, a twodimensional background and a set of local two-dimensional backgrounds, respectively. (d) to (f): Differences between the original image and three Wiener filtered images shown in (a) to (c), respectively. ( $g$ ) to (i): Fourier transforms of the corresponding differences in the middle row. A normal Wiener filter with a radial background fails to extract structural information, while a local 2D Wiener filter extracts all periodic information. Thus, the difference image ( $3 f$ ) does not show any structural features.
A periodic mask is usually binary, namely taking a value of one at diffraction spots and zero elsewhere. We may be able to modify the simple periodic mask to a Wiener type mask, where the background $\left|\hat{F}_{b}\right|$ in Eq. (1) is estimated from an area surrounding each diffraction spot.

\section{Examples}

We applied Wiener filters with various backgrounds mentioned above to a regular crystal of $\mathrm{Si}_{3} \mathrm{~N}_{4}$, and two non-ideal crystals of crysotile, a natural mineral of cylindrical structure, and $\mathrm{TiO}_{2}$ nanoparticles in amorphous $\mathrm{SiO}_{2}$ as shown below.

Example 1: $\mathrm{Si}_{3} \mathrm{~N}_{4}$

Here, we apply a Wiener filter to a regular crystal of $\mathrm{Si}_{3} \mathrm{~N}_{4}$ (Fig. 1a). Since its Fourier transform yields a regular periodic diffraction pattern (Fig. 1d), even a Wiener filter using a radial background gives a satisfactory result (Fig. 1b), while a Wiener filter, using a periodic mask background, gives a stronger filtering effect (Fig. 1c). The periodic structure emphasized at the top left part of the image may reflect the original structure before degradation from radiation damage. The differences between the original image and the two Wiener filtered images (Fig. 1e and 1f) show random structure only, which demonstrates that an extraction of the structure information is satisfactory.

\section{Example 2: Crysotile}

Although the radial or periodic mask Wiener filters work well for an ideal crystal image as shown for $\mathrm{Si}_{3} \mathrm{~N}_{4}$, they will not work for non-ideal crystals. Here, we will show a distinct example, for which a local $2 \mathrm{D}$ background is essential for a Wiener filter. Our sample image is taken from crysotile, a clay mineral $\mathrm{Mg}_{3} \mathrm{Si}_{2} \mathrm{O}_{5}(\mathrm{OH})_{4}$, which is a natural multilayered nanotube. An HREM image of this sample prepared by ion milling is shown in Fig. 2a. Here, the interlayer spacing is about $0.72 \mathrm{~nm}$, and a repeat distance of structural blocks within each layer is about $0.46 \mathrm{~nm}$. Thus, its structural information appears approximately on the circles in Fourier space as shown in Fig. 2b. Crysotile is extremely radiation sensitive, and easily becomes amorphous during observation. Thus, an image feature from the crystal is typically low contrast, and it is rather difficult to analyze a stacking structure from an original image.

Figure 3 shows three Wiener filtered images using (a) a radial background, (b) a two-dimensional background and (c) a set of two-dimensional backgrounds locally estimated over $64 \times 64$ pixels. The difference between the original image and the filtered image with the radial background clearly demonstrates an insufficient extraction of the structure 


\section{Why have 1,000 scientists} in $\mathbf{2 5}$ countries selected Minus $\mathbf{K}^{*}$ vibration isolators?

Because Minus $\mathrm{K}$ vibration isolation systems deliver $10 x$ to $100 x$ better performance than high-performance air systems, without air, and for air table prices.

\section{Visit www.minusk.com for more Information...}

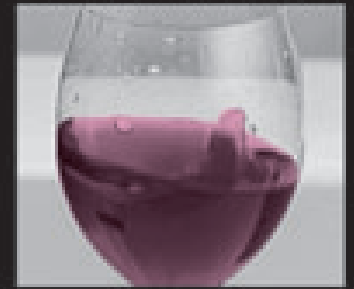

Without Minus $\mathbf{K}$

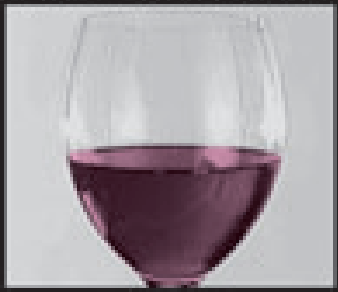

Whth Minus $\mathbf{K}$
VIBRATION ISOLATION BY:

\section{minus $\mathbf{k}^{\circ}$ TECHNOLOGY}

420 S. Hindry Ave., Unit E * Inglewood, CA 90301 USA Phone: 310-348-9656 * Fax: 310-348-9638 salescminusk.com * www.minusk.com

\section{PELCO ${ }^{\circledR}$ Silicon Nitride Membranes}

Next Generation $\mathrm{Si}_{3} \mathrm{~N}_{4}$ TEM Support Films with many advantages:

- Durable and chemically inert planar $50 \mathrm{~nm}$ substrate

- $3.0 \mathrm{~mm}$ circular frame compatible with standard TEM holders

- EasyGrip ${ }^{\text {TM }}$ micro rough edges for ease of handling

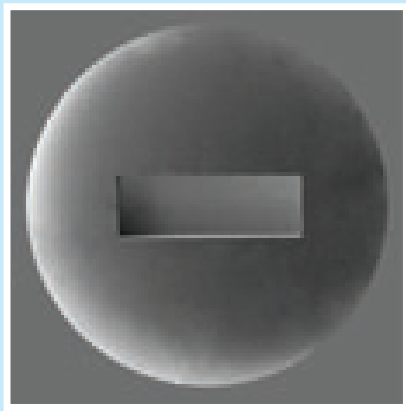

- Free from debris - no broken edges

- Large area support film: up to $0.5 \times 1.5 \mathrm{~mm}$

- Complimented with Aperture Frames and Blank Disks for nanotech experiments

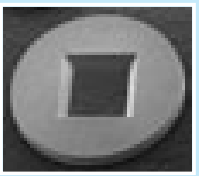
Aperture Frame

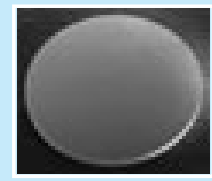

Blank Disks
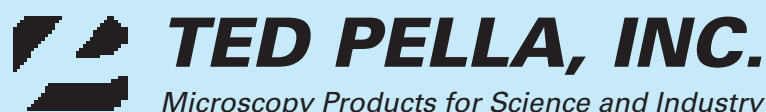

Microscopy Products for Science and Industry

\section{Less Hardware.} More Image. No Rival.

\section{The New Dual Standard.}

Our Elion" and $3 \mathrm{Max}$ "two-in-one detection platforms bring powerful new applications to leading dual beam, FIB and SEM systems.

Clion "ion mode (left) and electron mode (right) $950 \mathrm{pA}$ FIB current

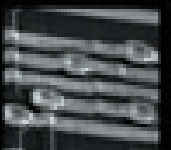

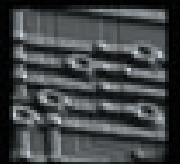

3 Max ${ }^{*}$ enhanced SE 3 image (left) and in-lens image (right) with other wibe identical condtions.
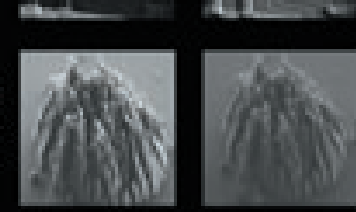

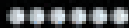

\section{9.}

g.

g.

http://www.el-mul.com

El-Mul

Electron \& Ion

Detection Solutions

You can detect the difference. 
(Fig. 3d). This insufficient extraction of structure information is also seen in the Fourier transform of the difference (Fig. 3g), which shows considerable amount of the signal information appearing on the concentric rings was left behind. This is because the radial background at the strong ring positions becomes higher than the normal background level.

The Wiener filtered image using a two-dimensional background gives substantial improvement for extracting the structure, and its difference from the original image shows only a faint feature (Fig. 3e). It is interesting to note that the Fourier transform of the difference (Fig. 3h) shows dark rings contrary to the case of the radial background (Fig. $3 g$ ). This means that most of the information, including noise, was passed (transferred) to the filtered image, since the Wiener mask $M_{w}$ is high at strong intensity over the rings. Thus, the faint feature comes from the damaged layer/random noise, whose spectrum passes through the mask.

The Wiener filtered image using the local two-dimensional background (Fig. 3c) shows structural feature more clearly and is smoother than Fig. 3b. It may be noted that the difference image (Fig. 3f) and its Fourier transform (Fig. 3i) no evidence of structural features. This indicates that the use of a local two-dimensional background is essential for this kind of structure.

Example 3: $\mathrm{TiO}_{2}$ nanoparticles in $\mathrm{SiO}_{2}$

We will show another remarkable example, for which a local $2 \mathrm{D}$ background is essential for a Wiener filter. Here, we choose $\mathrm{TiO}_{2}$ nanoparticles as a non-ideal crystal embedded in amorphous $\mathrm{SiO}_{2}$ (Fig. 4a). A thin film of $\mathrm{SiO}_{2}-\mathrm{TiO}_{2}$ (5:1) was formed on a silicon substrate by sol-gel processing. Then, $\mathrm{TiO}_{2}$ (anatase) nano-crystals were grown in the $\mathrm{SiO}_{2}$ matrix by hot-water treatment (Courtesy of A. Matsuda). Since nano-particles are oriented randomly as seen
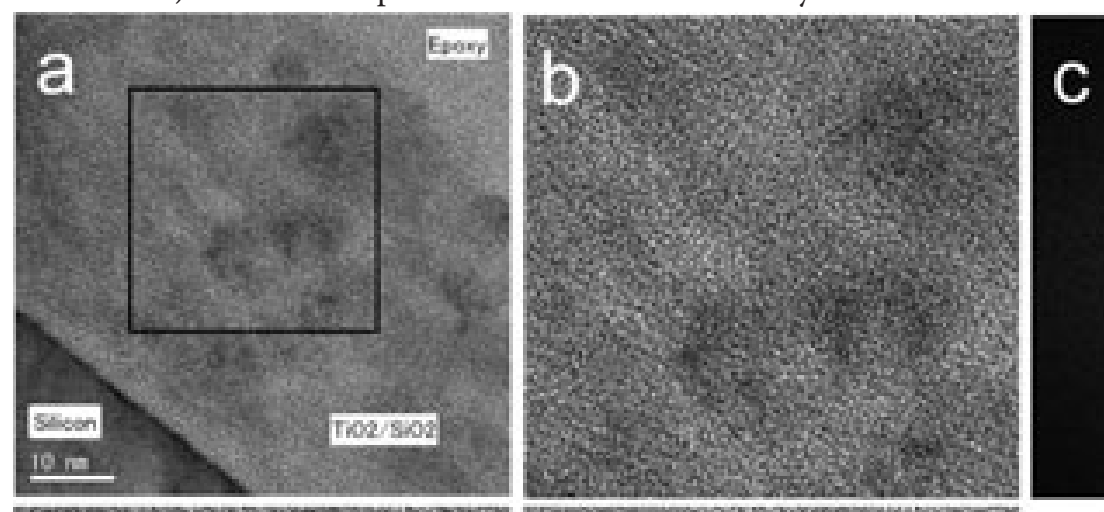

$$
\hat{F}_{D}=M_{D} F_{o}=\frac{\left|F_{o}\right|-\left|\hat{F}_{b}\right|}{\left|F_{o}\right|} F_{o}=\left(\left|F_{o}\right|-\left|\hat{F}_{b}\right|\right) e^{i \phi_{o}},
$$

where $\phi_{o}$ is the phase of the observed signal $F_{o}$, and $\hat{F}_{b}$ is the estimate of the background as before. $\hat{F}_{D}$ is set to zero, if $\left|F_{o}\right|-\left|\hat{F}_{b}\right| \leq 0$. The Difference filter can be applicable to a non-ideal crystal, when we use the new backgrounds developed here. Although Kilass wrote that the Wiener filter and Difference filter give comparable results, we found that the Wiener filter is usually better at extracting a periodic structure.

\section{Conclusions}

We have extended the usability of the Wiener and Difference filters even to the cases where a translational periodicity is limited to the order of a nano-meter. We have demonstrated that these filters make structural features visibly discernible in the cases shown

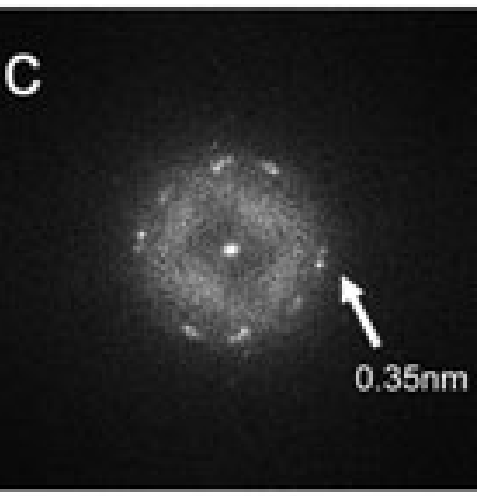
here. Without the detection of a structure by the operator, there is no way to analyze a structure quantitatively. Although filtering has been considered as a cosmetic process, we maintain that a careful filtering operation is an appropriate first step before beginning a quantitative analy-
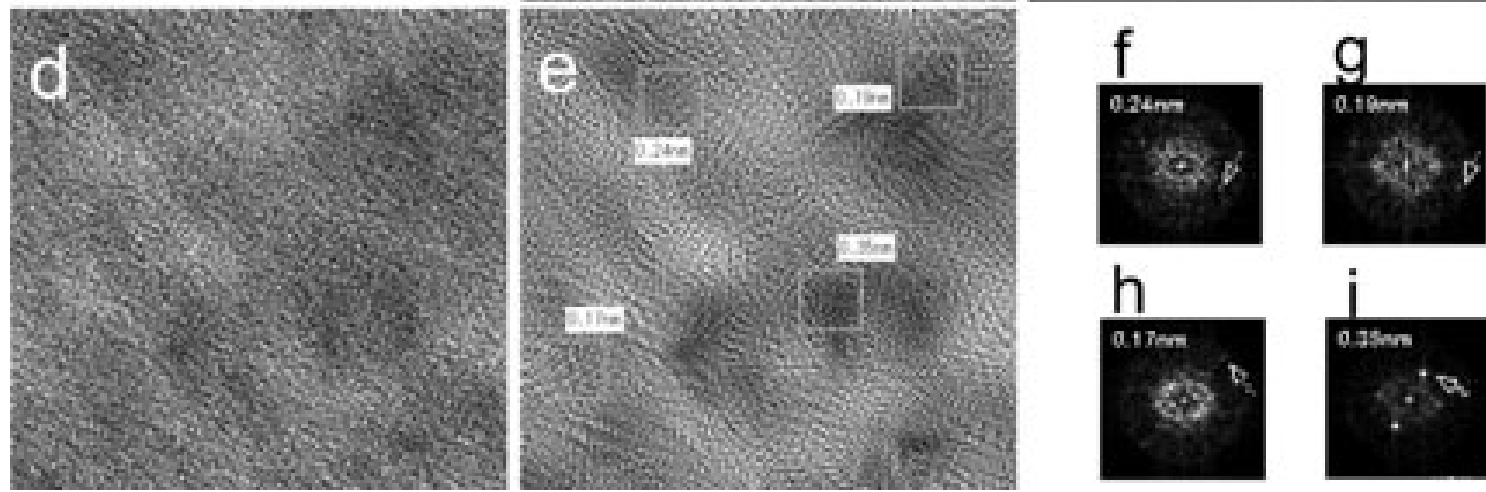

Figure 4 Wide sample view (a), of a selected area (512x512 pixels) to be processed (b), and its Fourier transform (c). Strong spots appear at the same distances from the origin. $(d)$ and (e): Two Wiener filtered images with radial and local two-dimensional backgrounds, respectively. The radial Wiener filter does not emphasize the periodic structures, while the local 2D Wiener filter makes nano-crystals substantially visible. $(f)$ to (i): Fourier transforms of small areas indicated in Fig. 4e showing fine spatial frequencies detected by using the local $2 D$ Wiener filter. sis.

\section{References}

[1] L.D. Marks, Ultramicroscopy 62 (1996) 43-52.

[2] R. Kilaas, J. Microscopy 190 (1997) 45-51.

[3] P.H.C. Eilers et al., Computational Statistics and Data Analysis 50 (2006) 61-76.

\section{Note to Readers}

Optimal Filters reported here are implemented as a plug-in, HREM Filters Pro, for DigitalMicrograph (Gatan Inc).

HREM Filters Lite is a freeware, and can be downloaded from the plug-ins page at: www.hremresearch.com. 


\section{Conquering the sub Ångstrom era}

Ultimate performance, stability, and flexibility - FEl's revolutionary Titan is the first and only platform designed to realize the full potential of corrected S/TEM imaging and analysis. With directly interpretable, sub Angstrom image resolution you will, quite literally, see things you've never seen before. Investigate property/structure relationships with atomic resolution. Clearly visualize the interfaces, discontinuities and crystalline defects that are often the key to material properties. Analyze elemental composition, chemical bonding states, electronic band gaps, and more with unprecedented spatial resolution. If you know S/TEM, you know this truly is a revolution.

If your research requires ultimate performance, and your success depends on generating ground breaking new results, call us. We'll be happy to give you an unfair advantage.

See more at fei.com 